\title{
European Society of Endocrinology Clinical Practice Guideline for long-term follow-up of patients operated on for a phaeochromocytoma or a paraganglioma
}

\author{
P F Plouin 1,2 , L Amar 1,2,8, O M Dekkers ${ }^{3,4}$, M Fassnacht ${ }^{5,6}$, A P Gimenez-Roqueplo ${ }^{2,7,8}$, \\ J W M Lenders ${ }^{9,10}$, C Lussey-Lepoutre ${ }^{8}$ and $O$ Steichen ${ }^{11,12}$ on behalf of the Guideline \\ Working Group ${ }^{+}$
}

\begin{abstract}
${ }^{1}$ Unité d'Hypertension Artérielle, Hôpital Européen Georges Pompidou, Assistance Publique-Hôpitaux de Paris, Université Paris-Descartes, F-75015 Paris, France, ${ }^{2}$ Faculté de Médecine, Sorbonne Paris Cité, Université ParisDescartes, F-75006 Paris, France, ${ }^{3}$ Department of Medicine, Division of Endocrinology, and Department of Clinical Epidemiology, Leiden University Medical Centre, Leiden, The Netherlands, ${ }^{4}$ Department of Clinical Epidemiology, Aarhus University Hospital, Aarhus, Denmark, ${ }^{5}$ Department of Internal Medicine I, Division of Endocrinology and Diabetology, University Hospital, University of Würzburg, Würzburg, Germany, ${ }^{6}$ Comprehensive Cancer Center Mainfranken, University of Würzburg, Würzburg, Germany, ${ }^{7}$ Assistance Publique-Hôpitaux de Paris, Hôpital Européen Georges Pompidou, Service de Génétique, F-75015 Paris, France, ${ }^{8}$ INSERM, UMR 970, Paris-Cardiovascular Research Center, F-75015 Paris, France, ${ }^{9}$ Department of Internal Medicine, Division of Vascular Medicine, Radboud University Medical Center, Nijmegen, The Netherlands, ${ }^{10}$ Department of Internal Medicine III, University Hospital Carl Gustav Carus, Technische Universität Dresden, Dresden, Germany, ${ }^{11}$ Assistance Publique-Hôpitaux de Paris, Sorbonne Universités, UPMC University Paris 06, Tenon Hospital, Internal Medicine Department, F-75020 Paris, France and ${ }^{12}$ INSERM, UMR_S1142, F-75006 Paris, France

${ }^{\dagger}$ (Details of this group are presented in the Methods section entitled Guideline Working Group)
\end{abstract}

Correspondence should be addressed to P F Plouin

Email pierre-francois.plouin@ aphp.fr

\begin{abstract}
Phaeochromocytomas and paragangliomas (PPGLs) are rare neuroendocrine tumours. Standard treatment is surgical resection. Following complete resection of the primary tumour, patients with PPGL are at risk of developing new tumoural events. The present guideline aims to propose standardised clinical care of long-term follow-up in patients operated on for a PPGL. The guideline has been developed by The European Society of Endocrinology and based on the Grading of Recommendations Assessment, Development and Evaluation (GRADE) principles. We performed a systematic review of the literature and analysed the European Network for the Study of Adrenal Tumours (ENS@T) database. The risk of new events persisted in the long term and was higher for patients with genetic or syndromic diseases. Follow-up in the published cohorts and in the ENS@T database was neither standardised nor exhaustive, resulting in a risk of follow-up bias and in low statistical power beyond 10 years after complete surgery. To inform patients and care providers in this context of low-quality evidence, the Guideline Working Group therefore prepared recommendations on the basis of expert consensus. Key recommendations are the following: we recommend that all patients with PPGL be considered for genetic testing; we recommend assaying plasma or urinary metanephrines every year to screen for local or metastatic recurrences or new tumours; and we suggest follow-up for at least 10 years in all patients operated on for a PPGL. High-risk patients (young patients and those with a genetic disease, a large tumour and/or a paraganglioma) should be offered lifelong annual follow-up.
\end{abstract}




\section{Overview of recommendations}

The recommendations are worded as either recommend (strong recommendation) or suggest (weak recommendation). We aimed to grade formally only the evidence underlying long-term follow-up. The strength/quality of evidence behind the recommendations is classified as very low $(\oplus \bigcirc \bigcirc \bigcirc)$, low $(\oplus \oplus \bigcirc \bigcirc)$, moderate $(\oplus \oplus \oplus \bigcirc)$ or strong $(\oplus \oplus \oplus \oplus)$. See Section 3.3.

\subsection{Diagnosis of malignancy}

$\mathrm{R}$ 1.1. We recommend defining malignancy of phaeochromocytoma and paraganglioma (PPGL) as the presence of metastasis in lymph node or other distant sites.

$\mathrm{R}$ 1.2. We suggest screening for metastatic tumours by $\left[{ }^{18} \mathrm{~F}\right]$-fluorodeoxyglucose positron emission tomography/ computed tomography (FDG PET/CT), if possible preoperatively: in patients with paragangliomas; in patients with phaeochromocytomas and elevated (i.e. above the reference) levels of 3-methoxytyramine (3MT) in plasma or urine; and in patients carrying germline mutations of the $S D H B$ gene.

\subsection{Perioperative workup}

$\mathrm{R}$ 2.1. We recommend that all patients with PPGL be considered for genetic testing.

$\mathrm{R}$ 2.2. We suggest assaying chromogranin A preoperatively in patients with normal preoperative plasma or urinary levels of metanephrine and normetanephrine (MN) and $3 \mathrm{MT}$.

$\mathrm{R}$ 2.3. We recommend assaying plasma or urinary levels of MN and 3MT 2-6 weeks after recovery from surgery in patients who had elevated MN levels preoperatively.

$\mathrm{R}$ 2.4. We suggest assaying chromogranin A levels 2-6 weeks after recovery from surgery in patients with normal preoperative $\mathrm{MN}$ and 3MT levels and elevated preoperative chromogranin A levels.

$\mathrm{R}$ 2.5. We recommend performing an imaging test 3 months after presumably complete surgery in patients operated on for PPGL who have elevated MN or 3MT postoperatively, in patients in whom $\mathrm{MN}$ and 3MT were normal preoperatively and in patients in whom MN and 3MT were not measured preoperatively.

\subsection{Duration of follow-up}

$\mathrm{R}$ 3.1. We suggest follow-up for at least 10 years in all patients operated on for a PPGL to screen for local or metastatic recurrences or new tumours. High-risk patients (young patients and those with a genetic disease, a large tumour and/or a paraganglioma) should be offered lifelong annual follow-up. $(\oplus \bigcirc \bigcirc \bigcirc)$

\subsection{Monitoring methods}

$\mathrm{R}$ 4.1. We recommend assaying plasma or urinary $\mathrm{MN}$ and 3MT every year to screen for local or metastatic recurrences or new tumours. $(\oplus \bigcirc \bigcirc \bigcirc)$

$\mathrm{R}$ 4.2. We suggest assaying plasma chromogranin A levels every year in patients operated on for MN-negative, 3MT-negative and chromogranin A-positive PPGL to screen for local or metastatic recurrences or new tumours. $(\oplus \bigcirc \bigcirc \bigcirc)$

$\mathrm{R} 4$.3. We suggest performing imaging tests every $1-2$ years in patients with biochemically inactive PPGL to screen for local or metastatic recurrences or new tumours. $(\oplus \bigcirc \bigcirc \bigcirc)$

\section{Overview of the condition}

\subsection{PPGLS}

PPGL are rare neuroendocrine tumours that arise from neural crest-derived cells of the sympathetic and parasympathetic nervous systems $(1,2)$ : phaeochromocytomas arise from the adrenal medulla and paragangliomas from the paraganglia outside the adrenal medulla (2). Paragangliomas are found in the abdomen, pelvis, thorax, head and neck and are classified as sympathetic or parasympathetic, depending on their origin. Phaeochromocytomas and most thoraco-abdomino-pelvic paragangliomas are of sympathetic origin. In most PPGL, chromaffin cells produce one or more catecholamines that are metabolised into MN or 3MT $(1,3)$. Most parasympathetic paragangliomas are found in the head or neck and do not usually produce catecholamines. Currently, a genetic germ line cause can be identified for around $40 \%$ of PPGL (4).

\subsection{Malignancy in PPGL}

At least $10 \%$ of phaeochromocytomas and sympathetic paragangliomas are malignant, although rates of malignancy differ according to the hereditary background $(1,4$, $5,6,7,8,9,10)$. Unlike for most tumours, no molecular, cellular or histological markers are available for determining whether a PPGL is malignant $(1,2,6)$. Therefore, malignancy is defined as the presence of metastasis at 
presentation or during follow-up, a metastasis being defined as the presence of chromaffin tissue in nonchromaffin organs $(1,4,5,6,7,8,9,10)$. The most frequent sites for metastases are lymph nodes, the skeleton, liver and lungs (7).

\subsection{New events in PPGL}

Following resection of the primary tumour, patients with PPGL are at risk of tumour persistence and of new tumoural events. Tumour persistence is the consequence of incomplete tumour resection, particularly in cases of malignant primary tumour and head and neck paragangliomas, or of tumour spillage during surgery $(11,12)$. New tumoural events are recurrences, defined as the reappearance of disease after complete tumour eradication, or new tumours $(8,13,14,15)$. Recurrences may be local (at the site of the primary tumour) or metastatic. New tumours are phaeochromocytomas or paragangliomas that arise in the contralateral adrenal or in a previously unaffected paraganglion. Local recurrence may arise from microscopic cells of the primary tumour that escaped the therapeutic intervention and later become detectable at the original site. However, persistence and recurrence are usually defined according to biochemical or imaging tests after the operation: if test results are positive, then the case shows persistence and if negative, recurrence $(6,8,16)$. In addition to new PPGL events, PPGL patients with syndromic diseases such as von Hippel-Lindau disease (VHL), multiple endocrine neoplasia type 2 (MEN2), neurofibromatosis 1 (NF1) or hereditary paragangliomas may develop nonPPGL tumours including renal cancer, medullary thyroid carcinoma or other cancers $(1,4)$.

\subsection{Long-term follow-up in PPGL}

The Endocrine Society USA, recently published a clinical practice guideline for the perioperative management of patients with PPGL (1). However, the long-term postoperative management was not addressed in detail. There is evidence that patients undergoing surgery for PPGL may develop new tumours or recurrence and that most recurrences are metastatic $(1,4,5,6,7,8,14,15,16)$. Although there are reports of the prognostic value of various clinical, biochemical, genetic, imaging and pathological features, there are no robust prognostic indices of tumour recurrence in patients with PPGL that can be used to guide clinical practice (8). The only exception is that the probability of new events seems to be higher in patients with inherited tumours who also are younger, have larger tumours and more frequently have multiple or extraadrenal tumours than patients with sporadic tumours $(4,6,7,8,16)$. There have been no randomised studies addressing optimal follow-up, and many clinically relevant questions are unanswered thus far: the appropriate duration of follow-up remains unclear, as new events may occur decades after the initial surgery; the distribution of events between recurrence (local or metastatic) and new tumours is not known; and it is uncertain which tests should be used to detect and monitor recurrences.

\section{Methods}

\subsection{Guideline Working Group}

The Clinical Committee of The European Society of Endocrinology (ESE) and the Endocrine Hypertension Working Group (WG) of the European Society of Hypertension created a WG (hereafter called the Guideline WG) to assess the risk of new events and the need for follow-up in patients operated on for a PPGL. The European Network for the Study of Adrenal Tumours (ENS@T) agreed to participate. Core participants were L Amar (Endocrinology, Paris), O M Dekkers (Clinical Epidemiology, Leiden), M Fassnacht (Endocrinology, Wurzburg), A P Gimenez-Roqueplo (Genetics, Paris), J Lenders (Internal Medicine, Nijmegen), C LusseyLepoutre (Nuclear Medicine, Paris), P F Plouin (Internal Medicine, Paris) and O Steichen (Internal Medicine and Medical Informatics, Paris). Additional WG members were A Januszewicz (Cardiology, Warsaw), M Mannelli (Endocrinology, Florence), H J L M Timmers (Internal Medicine, Nijmegen), B Wangberg (Surgery, Goteborg), A Wilson (Head of PheoParaTroopers, Kokomo, IN, USA) and F Zinzindohoué (Surgery, Paris).

\subsection{Aims of the guideline and target group}

The present guideline aims to contribute to improving knowledge and standardising clinical care regarding longterm follow-up of patients operated on for a PPGL and to strengthen cooperation between patients and care providers. The relevant carers include endocrinologists, internists, oncologists, surgeons, vascular medicine specialists, radiologists, geneticists, biologists, pathologists, general practitioners and patient organisations. The present guideline complements the clinical practice guideline recently published by The Endocrine Society (1). 


\subsection{Method for guideline development}

The methods used for the development of this guideline has been described previously (17). In short, the guideline was established on the basis of the Grading of Recommendations Assessment, Development and Evaluation (GRADE) principles, a systematic approach for reviewing evidence and grading recommendations $(17,18)$. The following elements were taken into account in the final recommendations: i) quality of the evidence and ii) values and preferences (patient preferences, goals for health, costs, management inconvenience, feasibility, etc.). The recommendations are worded as recommended (strong recommendation) or suggested (weak recommendation). The weighting of a strong recommendation can be stated as follows: reasonably informed persons (clinicians, patients and politicians) would want management to be in accordance with the recommendation. For a weak recommendation, most individuals would act in accordance with the guideline, but a substantial number would not. The strength (or quality) of the evidence underlying the recommendations is classified as very low $(\oplus \bigcirc \bigcirc \bigcirc)$, low $(\oplus \oplus \bigcirc \bigcirc)$, moderate $(\oplus \oplus \oplus \bigcirc)$ or strong $(\oplus \oplus \oplus \oplus)$. The only evidence formally graded is that underlying long-term follow-up (recommendations 3.1., 4.1., 4.2. and 4.3); the evidence for all other recommendations is ungraded.

There is no automatic step from evidence to recommendations. Available evidence is often of poor quality such that strong recommendations would be inappropriate, and standardised assessment of the level of evidence does not eliminate the need for clinical judgment. Also, recommendations are required in some situations even if there is no evidence, as treatment decisions have to be made. To optimise transparency, all recommendations provided are accompanied by text explaining why they are made.

\subsection{Systematic review of the literature}

PubMed and Embase from 1980 to 2011 were searched to review systematically all data relevant to the incidence of and factors associated with recurrences or new tumours after complete resection of phaeochromocytomas and thoraco-abdomino-pelvic paragangliomas. The review included 38 cohorts (published in English) of patients with these tumours and complete tumour resection, postoperative follow-up exceeding 1 month and recurrence or new tumours documented by pathology, hormonal and/or imaging tests. Details will be provided in a forthcoming article (19).

\subsection{ENS@T database compilation}

The presence or absence of a germline mutation in one of the PPGL susceptibility genes is a candidate predictor of long-term outcome (see Section 2.4). As many putatively relevant genes have been described only recently $(4,9)$, many of the published papers, from 1980 to the present, do not consider the presence or absence of inherited diseases. Most patients in the existing ENS@T PPGL database have been screened for the major known inherited diseases involving PPGL. The Guideline WG therefore obtained the approval of Felix Beuschlein (Division of Endocrine Research, University Hospital Munich, Germany), Chair of the ENS@T Executive Committee and of the members of the ENS@T PPGL working group, to compile the ENS@T database and use it to complement the review of the literature.

Data from six centres and 1153 patients were compiled including 701 with: i) resection of the primary tumour, ii) no evidence of persistent disease at postoperative assessment and iii) documented follow-up of 6 months or more. Details will be published later.

\subsection{External review}

The Guideline WG prepared a draft that was presented at the ESE Annual meeting in Dublin in May 2015, sent for revision and comments to $\mathrm{G}$ Eisenhofer and $\mathrm{R}$ de Krijger for advice specifically about biomarkers and pathology and displayed on the official website of the ESE to invite ESE members for comments.

\subsection{Clinical questions}

A non-systematic review presented at the third meeting of the Phaeochromocytoma and Paraganglioma Research Support Organization (8) was used to establish a set of questions that were circulated to and approved by the Guideline WG. The following questions were selected for a systematic review of the literature:

What is the incidence of recurrence (local or metastatic) or new tumours in patients operated on for PPGL with apparently complete tumour resection?

Which factors are associated with documented recurrence (local or metastatic) or new tumours in patients operated on for PPGL with apparently complete tumour resection? 


\section{Summary and interpretation of the evidence}

\subsection{Systematic review}

The results of the literature search will be presented in a forthcoming article (19). In total, 42 articles relevant to phaeochromocytomas and thoraco-abdomino-pelvic paragangliomas, corresponding to 38 cohorts, were analysed in detail. There were a total of 2509 patients, $53 \%$ were female and the mean age at diagnosis was 27-53 years. In total, 14 studies involved only patients with phaeochromocytomas; in the other 23 cohorts with information on tumour location, $89 \%$ of patients had a phaeochromocytoma. Four studies involved only patients with a syndromic disease (i.e. VHL, NF1 or MEN2); in the other 28 cohorts with phenotypic and/or genetic information, a syndromic or familial disease and/or a mutation in a gene predisposing to PPGL were documented in $27 \%$ of patients overall. A total of 2396 patients had undergone curative surgery.

Three factors were associated with an increased risk of recurrence in multivariate analysis in at least one study: syndromic disease, larger tumour and paraganglioma (vs phaeochromocytoma). None of the studies compared outcomes of follow-up with hormonal or imaging studies, or different follow-up intervals.

The pooled incidence rate of postoperative new events, based on data from 34 cohorts, was 0.95 (95\% CI: $0.68,1.25)$ per 100 person-years. Assuming a steady incidence over time, this converts into a 5 -year incidence of $4.7 \%$ (95\% CI: $4.0,6.1$ ), distributed as follows: new tumours $22 \%$, local recurrences $23 \%$ and metastatic recurrences $55 \%$. The incidence of new events was 0.87 (95\% CI: 0.59, 1.14) per 100 person-years in studies involving both syndromic and non-syndromic diseases, and 2.06 (95\% CI: 1.37, 2.75) per 100 person-years in studies involving only syndromic diseases $(P=0.005$ for the difference).

\subsection{ENS@T data}

The ENS@T cohort provided access to individual data and enabled univariate and multivariate analyses of candidate prognostic markers. The results will be published in a forthcoming article. In addition, it could be used to distinguishing between the three types of new events (local or metastatic recurrences or new tumours). The records from 701 patients who had no evidence of persistent disease at postoperative assessment after a
Table 1 Risk of new event and number of patients needed to screen (NNS) to detect 1 new event during the first 5 years following surgery.

\begin{tabular}{|c|c|c|}
\hline Group/subgroup & $\begin{array}{l}\text { Risk of new event } \\
\quad \%(95 \% \mathrm{Cl})\end{array}$ & $\begin{array}{c}\text { NNS } \\
n(95 \% \mathrm{Cl})\end{array}$ \\
\hline Overall & $10(8,14)$ & $10(7,13)$ \\
\hline \multicolumn{3}{|l|}{ Tumour location } \\
\hline Phaeochromocytoma & $8(6,11)$ & $12(9,18)$ \\
\hline $\begin{array}{l}\text { Thoraco-abdomino-pelvic } \\
\text { paraganglioma }\end{array}$ & $18(11,31)$ & $5(3,9)$ \\
\hline $\begin{array}{l}\text { Head and neck paraganglioma } \\
\text { Age at surgery }\end{array}$ & $25(11,57)$ & $4(2,9)$ \\
\hline$\geq 20$ years old at surgery & $9(7,12)$ & $11(8,15)$ \\
\hline$<20$ years old at surgery & $27(15,51)$ & $4(2,7)$ \\
\hline \multicolumn{3}{|l|}{ Phenotypic and genetic status } \\
\hline Non-syndromic/genetic disease & $7(5,11)$ & $13(9,21)$ \\
\hline Syndromic/genetic disease & $17(12,24)$ & $6(4,9)$ \\
\hline \multicolumn{3}{|l|}{ Tumour size } \\
\hline Tumour $<150 \mathrm{~mm}$ & $10(7,14)$ & $10(7,14)$ \\
\hline Tumour $\geq 150 \mathrm{~mm}$ & $26(6,100)$ & $4(1,15)$ \\
\hline
\end{tabular}

follow-up of 6 months or more were analysed. In total, $54 \%$ were women, $80 \%$ had at least one phaeochromocytoma and $34 \%$ had a genetic or syndromic disease. Median age at surgery was 46 years (interquartile range (IQR) 33, 57). Median tumour size was $44 \mathrm{~mm}$ (IQR 30, 60 ), and the tumour was larger than $50 \mathrm{~mm}$ in $44 \%$ of cases. Median follow-up was 54 months (IQR 25, 101). The risk of new events in the whole population was 10\% (95\% CI 8,14 ) over the first 5 years of follow-up (new tumours $42 \%$, local recurrences $13 \%$ and metastatic recurrences $45 \%)$. The risk of new events over the first 5 years of followup in selected subgroups is reported in the Table 1 . The incidence of new events did not decline after 5 years of follow-up, but estimates after 10 years of follow-up are imprecise due to the small numbers of patients for whom data were available.

\subsection{Conclusion and interpretation of the scientific literature}

In patients with PPGL who have undergone an apparently complete resection of the primary tumour, the risk of new events persists in the long-term and is higher for patients with genetic or syndromic diseases. However, follow-up was neither standardised nor exhaustive in the published cohorts or in the ENS@T database, resulting both in a risk of follow-up bias and in low statistical power beyond 10 years after complete surgery. The prognosis of patients still event-free 10 years after curative surgery remains uncertain, precluding strong recommendations beyond the first 10 years of follow-up. To inform patients and care 
providers in the context of low-quality evidence, the Guideline WG therefore prepared recommendations on the basis of expert consensus.

\section{Recommendations and rationale for the recommendations}

\subsection{Diagnosis of malignancy}

R. 1.1. We recommend defining malignancy in PPGL as the presence of metastasis in lymph nodes or other distant sites.

Rationale. No single clinical, biochemical or histological feature can distinguish malignant from benign PPGL $(1,2,6)$. The multi-parameter Phaeochromocytoma of the Adrenal Gland Scaled Score is not reliable (20) and the reproducibility of the recent Grading system for Adrenal Phaeochromocytoma and Paraganglioma (21) requires further validation. A metastasis is therefore defined as the presence of chromaffin tissue in a location where normally no chromaffin tissue is expected (see Section 2.2).

Remark 1. The proposed definition of malignancy relies on overt metastatic spread: it is specific but not sensitive. In the absence of apparent metastases, however, there are no data concerning prediction or prevention (e.g. adjuvant therapy) of metastatic spread.

Remark 2. This definition applies to metastatic primary tumours and to metastatic recurrences. Searching for metastases is an objective of the preoperative workup (1), but patients with primary metastatic tumours are outside of the scope of these guidelines. For the clinical course of subjects with documented metastatic PPGL, see references $(6,7)$.

$\mathrm{R}$ 1.2. We suggest screening for metastatic tumours by FDG PET/CT, if possible preoperatively: in patients with paragangliomas, in patients with phaeochromocytomas and elevated (i.e. above the upper limit of the reference range) levels of 3MT in plasma or urine, and in patients carrying germ line mutations of the $S D H B$ gene.

Rationale. As there is no specific biochemical marker of malignancy, the presence of metastases should be documented by imaging tests or by pathological investigations. Preoperative thoraco-abdomino-pelvic CT or magnetic resonance imaging may disclose metastases, or metastases may present as peritumoral lymph nodes or be confirmed during intra-operative exploration. Whole-body functional imaging can disclose distant metastases that are not seen on CT or MR imaging or during intra-operative exploration. Descriptions of several series have shown that malignant primary tumours are more frequently extraadrenal (paraganglioma rather than phaeochromocytoma) than apparently benign primary tumours (review in (8) and Section 4). High levels of 3MT are found in patients with malignant PPGL $(3,10)$. Primary malignant tumours and malignant recurrences are frequent in $S D H B$ mutation carriers and may also occur in $F H$ and MDH2 mutation carriers $(1,4,5,6,7,8,9)$. To document the tumoural status of patients with PPGL (patients with metastatic primary tumours or at risk of new postoperative events), we suggest performing CT or MR plus functional imaging in these three categories of high-risk patients.

Remark 1. There is evidence that FDG PET/CT scanning is superior to ${ }^{123}$ I-metaiodobenzylguanidine (mIBG) scintigraphy for detecting metastasis especially in patients with SDHB mutations (22), but the test is expensive. ${ }^{123} \mathrm{I}$-mIBG scintigraphy has a role in the identification of disease which can be treated with ${ }^{131}$ I-mIBG. New radiopharmaceuticals have been developed but raise issues of cost and availability that are not addressed here.

Remark 2. The determination of 3MT improves accuracy for diagnosing malignant PPGL $(3,10)$ but is not yet widely available.

\subsection{Perioperative workup}

$\mathrm{R}$ 2.1. We recommend that all patients with PPGL be considered for genetic testing.

Rationale. This recommendation is in The Endocrine Society practice guidelines for PPGL (1) because more than onethird of patients with PPGL have disease-causing germ line mutations in a PPGL susceptibility gene and because the presence of mutations has implications for the patient and her/his family (4). The systematic review of the literature and the compilation of the ENS@T database show that the risk of new events is about twice as high in patients with genetic or syndromic PPGL than in those with apparently sporadic disease (see Section 4). In addition, $S D H B$ mutation carriers are at high risk of malignant PPGL either at diagnosis or during follow-up $(4,5,6,7,8)$. Remark. The issues of diagnostic algorithms, possible negative consequences and financial costs of genetic testing need to be considered and are discussed in (1). 
R 2.2 We suggest assaying chromogranin A preoperatively in patients with normal preoperative plasma or urinary levels of MN and 3MT.

Rationale. The plasma concentration of chromogranin A may provide an alternative marker of functional activity in patients with PPGL and normal preoperative MN and 3MT levels. Note, however, that abdominal PPGL with normal preoperative $\mathrm{MN}$ are very rare, most PPGL with normal $\mathrm{MN}$ being head and neck paragangliomas.

$\mathrm{R}$ 2.3. We recommend assaying plasma or urinary levels of MN and 3MT 2-6 weeks after recovery from surgery in patients who had elevated MN levels preoperatively.

Rationale. The goal of postoperative testing is to document complete resection of the tumour. In patients operated on for $\mathrm{MN}$ - or 3MT-producing tumours, the presence of elevated (i.e. above the upper limit of the reference range) postoperative $\mathrm{MN}$ or 3MT levels strongly suggests that there is persistent disease. Such results should be followed by imaging tests to confirm the presence of, and locate, residual catecholamine-secreting tissue $(1,3,16)$.

Remark 1. Postoperative $\mathrm{MN}$ and 3MT determinations should be performed 2-6 weeks after surgery. Preanalytical sampling conditions and methods for determining $\mathrm{MN}$ and 3MT, preferably in the same assay, are considered in $(1,3,23)$.

Remark 2. As far as tumour resection has been complete as documented by normal postoperative MN or 3MT levels in $\mathrm{MN}$ - or 3MT-producing tumours, there are no data to justify different follow-up schemes in patients having undergone laparoscopic or open surgery.

$\mathrm{R}$ 2.4. We suggest assaying chromogranin A levels 2-6 weeks after recovery from surgery in patients with normal preoperative MN and $3 \mathrm{MT}$ levels and elevated preoperative chromogranin A levels.

Rationale. Chromogranin A plasma levels may serve an alternative biochemical marker in patients with PPGL and normal preoperative $\mathrm{MN}$ (see also $\mathrm{R} 4.2$ ).

$\mathrm{R}$ 2.5. We recommend performing an imaging test 3 months after presumably complete surgery in patients operated on for PPGL who had elevated MN or 3MT postoperatively, in patients in whom MN and 3MT were normal preoperatively and in patients in whom $\mathrm{MN}$ and 3MT were not measured preoperatively.
Rationale. Persistently elevated concentrations of $\mathrm{MN}$ and/or 3MT postoperatively suggest incomplete tumour resection, and imaging can be used for assessing persistent residual tumour mass (16). No alteration in $\mathrm{MN}$ or 3MT can be expected in patients who underwent surgery for a PPGL with normal MN and 3MT; imaging is the only useful follow-up test available for such patients. Postoperative assay of $\mathrm{MN}$ and 3MT plus an imaging test are required in patients in whom $\mathrm{MN}$ and 3MT were not measured preoperatively because they may belong to either of the first two categories of patients.

Remark. Follow-up imaging of all operated PPGL patients is not cost-effective, although imaging can be used as an adjunct to biochemical tests for individual patients with a very high suspicion of incomplete resection.

\subsection{Duration of follow-up}

$R$ 3.1. We suggest follow-up for at least 10 years in all patients operated on for a PPGL to monitor local or metastatic recurrences or new tumours. High-risk patients (young patients and those with a genetic disease, a large tumour and/or a paraganglioma) should be offered lifelong annual follow-up. $(\oplus \bigcirc \bigcirc \bigcirc)$.

Rationale. The incidence of new events is low, about one per 100 person-years, but more than $40 \%$ of new events are malignant recurrence, and news events may occur after a 5-year event-free period (see Section 4). The 5-year risk of new events is $27 \%$ if the size of the primary tumour exceeds $150 \mathrm{~mm}$, but there is no 'safe' tumour size below which there is no risk of a new event. Likewise, the 5-year risk of a new event is $27 \%$ in young patients $(<20$ years old) and around 20-25\% in those with paraganglioma, but older patients and those with phaeochromocytoma still have a 5-year risk of new events of around 10\%. The available data do not reveal any subgroup of patients with PPGL in which follow-up may be safely interrupted at any time (see Section 4). Patients operated on for PPGL should be provided with a personalised estimate of the risk of new events based on age, genetic test results and tumour site and size. The periodicity and duration of follow-up should take into account this risk estimate but also the psychological and financial burden of follow-up. High-risk patients (young patients and those with a genetic disease, a large tumour and/or a paraganglioma) should be offered lifelong annual follow-up. Quality of life is impaired in patients with PPGL (24), and psychological support should be offered to affected patients and their families. However, 
it should be noted that no diagnostic strategy has been proven beneficial in a RCT setting.

Remark. Additional disease-specific follow-up is required in patients with syndromic PPGL who are at risk of non-PPGL tumoural events (see Section 2.3).

\subsection{Monitoring methods}

$\mathrm{R}$ 4.1. We recommend assaying plasma or urinary $\mathrm{MN}$ and $3 \mathrm{MT}$ every year to screen for local or metastatic recurrence or new tumours. $(\oplus \bigcirc \bigcirc \bigcirc)$

Rationale. There is evidence that determination of plasma free or urinary fractionated $\mathrm{MN}$ is more sensitive than assaying catecholamine and vanillylmandelic acid for detection of PPGL $(1,3)$. There is cross-sectional evidence that higher than normal levels of 3MT are associated with malignancy in PPGL $(3,10)$. There is no evidence that MN and 3MT determinations allow earlier detection of recurrence or new tumours than imaging tests, but MN determination is widely available, harmless and cheap, and pre-analytical conditions are relatively simple (23).

Remark 1. Determining MN and 3MT only in patients who present with hypertension or adrenergic symptoms during follow-up is not sound because these signs and symptoms have poor sensitivity and specificity for predicting recurrence or new tumours (16).

Remark 2. Patients operated on for a biochemically inactive tumour can subsequently develop new biochemically active tumours, and this is particularly true of patients with hereditary disease $(1,25)$.

R 4.2. We suggest assaying plasma chromogranin A every year to screen for local or metastatic recurrence or new tumours in patients operated on for MN- and 3MTnegative and chromogranin A-positive PPGL. $(\oplus \bigcirc \bigcirc \bigcirc)$

Rationale. SDHB mutation carriers with PPGL may exhibit normal MN and elevated chromogranin A levels in plasma (26). Plasma chromogranin A has been reported to vary longitudinally with chemotherapy in patients with malignant phaeochromocytomas (27). However, the first report was cross-sectional and did not report 3MT levels, and the second did not report MN levels. It is possible that monitoring chromogranin A levels is an alternative biochemical monitoring test in patients with PPGL and normal $\mathrm{MN}$ and 3MT.

$\mathrm{R} 4$.3. We suggest performing imaging tests every $1-2$ years in patients with biochemically inactive PPGL to screen for local or metastatic recurrences or new tumours. $(\oplus \bigcirc \bigcirc \bigcirc)$

Rationale. Imaging tests are the only option in cases where there are no reliable biochemical markers. It is not known which imaging technique is best. To minimise radiation exposure, one option is to perform thoraco-abdominopelvic MR. Also, the optimal time interval for periodic follow-up imaging is unknown. There are no observational or randomised studies that support any particular time interval in this patient group. Consequently, the recommended time interval of 1-2 years is arbitrary.

Remark. This applies mostly to patients with head and neck paragangliomas.

\subsection{Specific conditions}

Pregnancy. Although pregnancy and delivery can trigger acute cardiovascular events in patients with PPGL (28), it is not known whether pregnancy alters tumour growth or the metabolism of catecholamines in these conditions. Tests intended to detect new events should be proposed to women who have been operated on for a PPGL and who plan to become pregnant. The above recommendations for long-term follow-up apply to patients of childbearing age.

Elderly and frail patients. Considering the absolute risks, see Section 4.2. and Table 1, screening for new events does not appear necessary for patients who have been operated on for PPGL and have a reduced life expectancy.

\section{Suggestions for future research}

Guidelines concerning long-term follow-up of patients operated on for a PPGL should be formulated according to the incidence of new events and the assessment of potential prognostic factors. The evidence collected for the present guidelines is preliminary and more information is needed, particularly as regards: the mean time from surgery to the first new event, the diagnostic performance and effectiveness of screening tests and the benefit of early diagnosis of asymptomatic new events.

Kaplan-Meier estimates of new events in sporadic and inherited PPGL syndromes are required, together with estimates of the distribution of events between metastatic recurrences and new tumours across these categories of tumours. These estimates could be provided by work with prospective cohorts of consecutive patients at risk of recurrence (i.e. with documented complete resection of 
the primary tumour) who have undergone comprehensive genetic testing and with few losses to follow-up. Such cohorts could be used to document the clinical, biochemical, imaging or tumour characteristics associated with recurrence and to identify how to tailor follow-up to individual patients. Given the low prevalence of PPGL, international collaborative studies and registries are required to confirm and improve guidelines for the follow-up of these patients.

\section{Declaration of interest}

The authors declare that there is no conflict of interest that could be perceived as prejudicing the impartiality of the research reported.

Funding

This work was supported by the European Society of Endocrinology.

\section{Acknowledgements}

We thank the following experts for their valuable and critical comments to this guideline: E Baudin (Oncology, Paris), A Grossman (Endocrinology, Oxford), C Jimenez (Oncology, Houston), J Widimsky (Internal Medicine, Prague) and W Young (Endocrinology, Rochester). Prior to publication, the guideline was also made available to ESE members for comment.

\section{References}

1 Lenders JW, Duh QY, Eisenhofer G, Gimenez-Roqueplo AP, Grebe SK, Murad MH, Naruse M, Pacak K \& Young WF Jr \& Endocrine Society. Pheochromocytoma and paraganglioma: an Endocrine Society Clinical Practice Guideline. Journal of Clinical Endocrinology and Metabolism 2014 99 1915-1942. (doi:10.1210/jc.2014-1498)

2 DeLellis RA, Lloyd RV, Heitz PU \& Eng C. 2004 World Health Organization classification of tumours. In Pathology and Genetics of Tumour of Endocrine Organs. Lyon: IARC Press, 147-158

3 Eisenhofer G \& Peitzsch M. Laboratory evaluation of pheochromocytoma and paraganglioma. Clinical Chemistry 201460 1486-1499. (doi:10.1373/clinchem.2014.224832)

4 Favier J, Amar L \& Gimenez-Roqueplo AP. Paraganglioma and phaeochromocytoma: from genetics to personalized medicine. Nature Reviews. Endocrinology 201511 101-111. (doi:10.1038/nrendo. 2014.188)

5 Gimenez-Roqueplo AP, Favier J, Rustin P, Rieubland C, Crespin M, Nau V, Khau Van Kien P, Corvol P, Plouin PF, Jeunemaitre X et al. Mutations in the SDHB gene are associated with extra-adrenal and/or malignant phaeochromocytomas. Cancer Research 200363 5615-5621.

6 Chrisoulidou A, Kaltsas G, Ilias I \& Grossman AB. The diagnosis and management of malignant phaeochromocytoma and paraganglioma. Endocrine-Related Cancer 200714 569-586. (doi:10.1677/ERC-07-0074)

7 Amar L, Baudin E, Burnichon N, Peyrard S, Silvera S, Bertherat J, Bertagna X, Schlumberger M, Jeunemaitre X, Gimenez-Roqueplo AP et al. Succinate dehydrogenase B gene mutations predict survival in patients with malignant pheochromocytomas or paragangliomas. Journal of Clinical Endocrinology and Metabolism 200792 3822-3828. (doi:10.1210/jc.2007-0709)
8 Amar L, Fassnacht M, Gimenez-Roqueplo AP, Januszewicz A, Prejbisz A, Timmers $\mathrm{H} \&$ Plouin PF. Long-term postoperative follow-up in patients with apparently benign pheochromocytoma and paraganglioma. Hormone and Metabolic Research 201244 385-389. (doi:10.1055/s-00311301339)

9 Cascón A, Comino-Méndez I, Currás-Freixes M, de Cubas AA, Contreras L, Richter S, Peitzsch M, Mancikova V, Inglada-Pérez L, PérezBarrios A et al. Whole-exome sequencing identifies MDH2 as a new familial paraganglioma gene. Journal of National Cancer Institute 2015 107 djv053.

10 Eisenhofer G, Lenders JW, Siegert G, Bornstein SR, Friberg P, Milosevic D, Mannelli M, Linehan WM, Adams K, Timmers HJ et al. Plasma methoxytyramine: a novel biomarker of metastatic pheochromocytoma and paraganglioma in relation to established risk factors of tumour size, location and SDHB mutation status. European Journal of Cancer 201248 1739-1749. (doi:10.1016/j.ejca.2011.07.016)

11 Capatina C, Ntali G, Karavitaki N \& Grossman AB. The management of head-and-neck paragangliomas. Endocrine-Related Cancer 201320 R291-R305. (doi:10.1530/ERC-13-0223)

12 Rafat C, Zinzindohoue F, Hernigou A, Hignette C, Favier J, Tenenbaum F, Gimenez-Roqueplo AP, Plouin PF \& Amar L. Peritoneal implantation of pheochromocytoma following tumour capsule rupture during surgery. Journal of Clinical Endocrinology and Metabolism 201499 E2681-E2685. (doi:10.1210/jc.2014-1975)

13 Miller AB, Hoogstraten B, Staquet M \& Winkler A. Reporting results of cancer treatment. Cancer 198147 207-214. (doi:10.1002/10970142(19810101)47:1 <207::AID-CNCR2820470134>3.0.CO;2-6)

14 Asari R, Scheuba C, Kaczirek K \& Niederle B. Estimated risk of pheochromocytoma recurrence after adrenal-sparing surgery in patients with multiple endocrine neoplasia type 2A. Archives of Surgery 2006141 1199-1205. (doi:10.1001/archsurg.141.12.1199)

15 Chen H, Sippel RS, O’Dorisio MS, Vinik AI, Lloyd RV \& Pacak K \& North American Neuroendocrine Tumour Society (NANETS). The North American Neuroendocrine Tumour Society consensus guideline for the diagnosis and management of neuroendocrine tumours: pheochromocytoma, paraganglioma, and medullary thyroid cancer. Pancreas 201039 775-783. (doi:10.1097/MPA.0b013e3181ebb4f0)

16 Plouin PF, Chatellier G, Fofol I \& Corvol P. Tumour recurrence and hypertension persistence after successful pheochromocytoma operation. Hypertension 199729 1133-1139. (doi:10.1161/01.HYP. 29.5.1133)

17 Dekkers OM \& Burman P \& ESE Clinical Committee. ESE guidelines, why and how. European Journal of Endocrinology 2015173 E1-E2. (doi:10.1530/EJE-15-0625)

18 Guyatt G, Oxman AD, Akl EA, Kunz R, Vist G, Brozek J, Norris S, FalckYtter Y, Glasziou P, DeBeer H et al. GRADE guidelines: 1. IntroductionGRADE evidence profiles and summary of findings tables. Journal of Clinical Epidemiology 201164 383-394. (doi:10.1016/j.jclinepi. 2010.04.026)

19 Amar L, Lussey-Lepoutre C, Lenders JWM, Djadi-Prat J, Plouin P-F \& Steichen O. MANAGEMENT OF ENDCRINE DISEASE: Recurrence or new tumors after complete resection of phaeochromocytomas and paragangliomas: a systematic review and meta-analysis. European Journal of Endocrinology 2016 In press. (doi:10.1530/EJE-16-0189)

$20 \mathrm{Wu}$ D, Tischler AS, Lloyd RV, DeLellis RA, de Krijger R, van Nederveen F \& Nosé V. Observer variation in the application of the Pheochromocytoma of the Adrenal Gland Scaled Score. American Journal of Surgical Pathology 200933 599-608. (doi:10.1097/PAS. Ob013e318190d12e)

21 Kimura N, Takayanagi R, Takizawa N, Itagaki E, Katabami T, Kakoi N, Rakugi H, Ikeda Y, Tanabe A, Nigawara T et al. Pathological grading for predicting metastasis in phaeochromocytoma and paraganglioma. Endocrine-Related Cancer 201421 405-414. (doi:10.1530/ERC-13-0530)

22 Rufini V, Treglia G, Castaldi P, Perotti G \& Giordano A. Comparison of metaiodobenzyl-guanidine scintigraphy with positron emission 
tomography in the diagnostic work-up of pheochromocytoma and paraganglioma: a systematic review. Quarterly Journal of Nuclear Medicine and Molecular Imaging 201357 122-133.

23 Willemsen JJ, Ross HA, Lenders JW \& Sweep FC. Stability of urinary fractionated metanephrines and catecholamines during collection, shipment, and storage of samples. Clinical Chemistry 200753 268-272. (doi:10.1373/clinchem.2006.075218)

24 van Hulsteijn LT, Kaptein AA, Louisse A, Smit JW \& Corssmit EP. Avoiding and nonexpressing: coping styles of patients with paragangliomas. Journal of Clinical Endocrinology and Metabolism 201398 3608-3614. (doi:10.1210/jc.2013-1340)

25 Gimenez-Roqueplo AP, Caumont-Prim A, Houzard C, Hignette C, Hernigou A, Halimi P, Niccoli P, Leboulleux S, Amar L, Borson-Chazot F et al. Imaging work-up for screening of paraganglioma and pheochromocytoma in SDHx mutation carriers: a multicenter prospective study from the PGL.EVA Investigators. Journal of Clinical Endocrinology and Metabolism 201398 E162-E173. (doi:10.1210/jc. 2012-2975)

26 Zuber S, Wesley R, Prodanov T, Eisenhofer G, Pacak K \& Kantorovich V. Clinical utility of chromogranin A in SDHx-related paragangliomas. European Journal of Clinical Investigation $2014 \mathbf{4 4} 365-371$. (doi:10.1111/eci.12245)

27 Rao F, Keiser HR \& O'Connor DT. Malignant pheochromocytoma. Chromaffin granule transmitters and response to treatment. Hypertension 200036 1045-1052. (doi:10.1161/01.HYP.36.6.1045)

28 Oliva R, Angelos P, Kaplan E \& Bakris G. Pheochromocytoma in pregnancy: a case series and review. Hypertension 201055 600-606. (doi:10.1161/HYPERTENSIONAHA.109.147579)

Received 9 November 2015

Revised version received 15 January 2016

Accepted 19 January 2016 